\title{
Changes in Cardiac Levels of Caspase-8, Bcl-2 and NT-proBNP Following 4 Weeks of Aerobic Exercise in Diabetic Rats
}

\author{
Saeid Tanoorsaz ${ }^{1}$, Naser Behpoor ${ }^{2 *}$, Vahid Tadibi ${ }^{3}$ \\ 1Ph.D Student in Exercise Physiology, Faculty of Physical Education and Sport Sciences, Razi University of Kermanshah, \\ Kermanshah, Iran \\ ${ }^{2}$ Assistant Professor of Exercise Medicine, Faculty of Physical Education and Sport Sciences, Razi University of Kermanshah, \\ Kermanshah, Iran \\ ${ }^{3}$ Associate Professor of Exercise Physiology, Faculty of Physical Education and Sport Sciences, Razi University of Kermanshah, \\ Kermanshah, Iran
}

\section{*Correspondence to \\ Naser Behpoor, Assistant Professor of Exercise Medicine, Faculty of Physical Education and Sport Sciences, Razi University of Kermanshah, Kermanshah, Iran. Tel: +98 8334283272; \\ Email:n_behpoor@yahoo.com}

Received September 23, 2017 Accepted October 29, 2017 Published online December 31, 2017

\begin{abstract}
Introduction: Cardiac apoptosis is one of the most important cardiovascular complications of diabetes. We aimed to investigate the changes of caspase-8, Bcl-2, and $\mathrm{N}$-terminal pro B-type natriuretic peptide (NT-proBNP) in cardiac tissue after 4 weeks of aerobic exercise in male rats with diabetes.

Methods: Forty adult male rats were randomly allocated to healthy control, diabetes, control + exercise and exercise + diabetes groups. Diabetes was induced by intraperitoneal injection of streptozotocin (STZ) solution (55 mg/kg). Two weeks after injection, fasting blood glucose levels were measured. After the induction of diabetes, the exercise program was performed for 4 weeks ( 5 sessions per week) at a speed of 15 to $18 \mathrm{~m} / \mathrm{min}$ for 25 to 44 minutes. Forty-eight hours after the last training session, the subjects were anesthetized and the heart muscle was removed. Caspase-8, Bcl-2 and NT-proBNP levels were measured by ELISA method.

Results: The induction of diabetes in the control group resulted in a significant increase in caspase-8, and NT-proBNP levels while an insignificant increase was observed for Bcl-2 levels $(P<0.05)$. In non-diabetic groups, exercise caused no changes in caspase-8, NT-proBNP and Bcl-2 $(P<0.05)$. Exercise in diabetic groups significantly decreased NT-proBNP while no changes were observed in caspase-8 and Bcl-2 $(P<0.05)$.

Conclusion: Our findings showed that diabetes increases the pro-apoptotic and anti-apoptotic agent. In addition, 4 weeks of regular aerobic exercises can be used as a non-pharmacological strategy to reduce the complications of apoptosis in diabetic cardiomyocytes.

Keywords: Apoptosis, NT-proBNP, Streptozotocin, Aerobic exercise
\end{abstract}

\section{Introduction}

Cardiovascular disorders are the main cause of morbidities and mortalities in diabetic patients, not only due to coronary artery disease and related high blood pressure but also due to direct adverse effects of diabetes on the heart and independent of other pathological factors. Research has shown that apoptosis plays a major role in the pathogenesis of diabetes-induced heart disease. ${ }^{1}$ Collagen is precipitated when heart cells are reduced by apoptosis. Ultimately, this reduction in cardiac compliance increases the tension of the heart muscle cell wall, causing ventricular dysfunction. The occurrence of apoptosis in the pathway of diabetes-induced myocardial damage has been proven by activating pathway components of apoptosis and caspase activity. In addition, the death of myocardial cells is known as a main contributor to the development of diabetes-induced cardiovascular damage. ${ }^{1-5}$ In fact, various studies have shown that diabetes significantly increases apoptosis in cardiac cells. ${ }^{5}$ In the early stages of heart

(C) 2017 The Author(s); Published by Zabol University of Medical Sciences. This is an open-access article distributed under the terms of the Creative Commons Attribution License (http://creativecommons.org/licenses/by/4.0), which permits unrestricted use, distribution, and reproduction in any medium, provided the original work is properly cited. 
damage, the number of cells that are lost is greater, which indicates the activation of the incremental regulation of anti-apoptotic pathways after the reduction of the cells. ${ }^{4}$ The process of apoptosis and planned cell death is regulated by some mitochondrial proteins including B-cell lymphoma-2 (Bcl-2) proteins, which are divided into 2 parts of the anti-apoptotic proteins (Bcl-2, Bcl$\mathrm{XL}, \mathrm{Bcl}-\mathrm{W}, \mathrm{Bfl}-1$ and Mcl-1) and pro-apoptotic proteins (Bax, Bak, Bad, Bcl-Xs, Bid, Bik, Bim and Hrk) which play a leading role in accelerating the onset of an attack. ${ }^{6}$ Whereas apoptosis is regulated by anti-apoptotic proteins by preventing the release of cytochrome $c$ from the mitochondria, pro-apoptotic proteins accelerate its release. $^{6}$

As a proto-oncogene antagonist of apoptosis at mitochondrial levels, with a weight of $28 \mathrm{kD}, \mathrm{Bcl}-2$ prevents the oxidative damage to the cell and is known as one of the most prominent inhibitors of apoptosis proteins, which, in addition to enhancing the release of cytochrome c from mitochondria, the integrity of the mitochondrial membrane by attaching $\mathrm{H}+$ ions to the apoptotic protease activating factor (apaf-1), prevents the activation of caspase-9.6, A mitochondrion is an inseparable component of the internal pathway of apoptosis and the site of deposition of many of the proteins interfering in the early stages of this process including the members of the Bcl-2 family. ${ }^{8}$ Mitochondrial functions are impaired as a result of DNA damage leading to irreversible cholesterol; therefore, Mitochondria participate in both the internal and external pathways of programmed cell death. ${ }^{9}$

In general, the pathways involved in stimulating the apoptosis process are divided into 2 categories as follows: internal pathway (or mitochondrial pathway), which is regulated by $\mathrm{Bcl}-2$ family proteins and by activating $\mathrm{Bak} / \mathrm{Bax}$, leads to the permeability of the mitochondrial membrane and the external pathway (or the pathway for death receptors), which is started following the inclusion of the TNF receptor ligand coating and is resulted in the activation of caspase- 8 and, consequently, caspase-3.,9 The process of apoptosis is carried out by a family of cysteine proteases called caspase. ${ }^{10}$ The external pathway of apoptosis begins through death receptors and activates caspase- 8 . After activation, caspase- 8 can directly activate the active caspases or act through Bid protein. ${ }^{11}$ In the internal pathway, the release of cytochrome $c$ from mitochondrion activates caspase- 9 and ultimately activates caspases. ${ }^{10}$ There is a close relationship between these two pathways so that Bid protein as caspase- 8 substrate releases cytochrome $\mathrm{c}$ after transfer to mitochondrion. ${ }^{13}$ Previous reports have noted that diabetes increases the levels and activity of caspase- $8 .{ }^{14}$ Studies also showed that the level of prohorrmone brain natriuretic peptide (NTproBNP) is significantly higher in diabetic subjects than in healthy subjects. ${ }^{15-17} \mathrm{BNP}$ levels have a significant positive correlation with cardiac failure and severity of hypertrophy and poor ventricular diastolic function. ${ }^{18-21}$ Researchers have argued that NT-proBNP level can be measured as a predictor of left ventricular malfunction and increased risk of death due to damage to the heart muscle..$^{22-25}$ Research has shown that regular exercises modify many metabolic disorders in the diabetic population in addition to their beneficial effects on systemic changes associated with obesity and type 2 diabetes., ${ }^{415}$ Such fluctuations are due to indirect effects of systemic alternations due to exercise and their direct effects on cardiac contractile activity during exercise. ${ }^{15}$ Exercise plays a protective role in the heart against complications of diabetes through the reduction of oxidative stress and apoptosis in heart cells. ${ }^{16}$ Due to a dearth of research on the effects of exercise on the adverse effects of diabetes on heart muscle damage and apoptosis, we examined the effects of a 4-week course of increasing aerobic exercise on cardiac markers of caspase-8, Bcl-2 and NT-proBNP in diabetic male rats.

\section{Methods}

Animals

Based on the model used in the study done by Chow et al in the selection of clinical trial samples, ${ }^{26} 40$ male Wistar rats weighing 200.63 \pm 17.47 were obtained from the Animal Breeding Center of Pasteur Institute of Iran-Tehran and transferred to the Animal Laboratory of Faculty of Physical Education and Sports Sciences of Mazandaran University. All subjects were kept under controlled environmental conditions with an average temperature of $22 \pm 3^{\circ} \mathrm{C}$, a 12-hour light cycle, a 12-hour cycle of darkness and free access to water and food. Mouse food was purchased from Behparvar Company, Iran.

\section{Allocation and Training Protocol}

The animals were randomly divided into 4 groups of 10 rats, ${ }^{26}$ including control, diabetes, control + exercise, and exercise + diabetes. Before performing the exercise protocol, the subjects were introduced to the treadmill for 1 week. The familiarization program consisted of 5 walking and running sessions at a speed of 5 to 8 $\mathrm{m} / \mathrm{s}$ without slope for 8 to 10 minutes. The training program included running on a non-slip treadmill with a progressive overload principle between 25 minutes in the first week and 44 minutes in the fourth week (one minute increase in training time per session compared to the previous session) and between $15 \mathrm{~m} / \mathrm{min}$ in the first week, up to $18 \mathrm{~m} / \mathrm{min}$ in the fourth week $(1 \mathrm{~m} / \mathrm{min} / \mathrm{wk})$, and 5 sessions per week for 4 weeks. ${ }^{27,28}$ To warm up, the subjects ran at $7 \mathrm{~m} / \mathrm{min}$ for 3 minutes at the beginning of each training session, and then to reach the desired speed, the speed of $2 \mathrm{~m} / \mathrm{min}$ for each minute was added to the treadmill. In order to cool the body in every training session, the speed of the treadmill decreased steadily until it reached the initial speed.

Diabetes Induction

Diabetes induction was done by an intraperitoneal 
injection of streptozotocin (STZ) solution from Sigma Aldrich Germany (CAS 18883-66-4-Calbiochem), soluble in citrate buffer $(\mathrm{pH}=4.5$ and $0.1 \mathrm{~mol}$ concentration) and $55 \mathrm{mg} / \mathrm{kg}$ body weight. ${ }^{29}$ Fourteen days after STZ injection, blood glucose concentration was measured using blood samples collected from animals with a glucometer. The criterion to be diabetic was the blood glucose level greater than $250 \mathrm{mg} / \mathrm{dL}$. For the control group, in order to equalize the effect of injection of $0.1 \mu \mathrm{m}$ citrate buffer, the same volume was injected intraperitoneally. ${ }^{17}$

\section{Caspase-8, Bcl-2, and pro-BNP Measurement}

Forty-eight hours after the last training session, all groups were anesthetized under completely similar conditions and fasting with intravenous injection of peritoneal ketamine (50 mg/kg body weight) and xylazine $(3 \mathrm{mg} /$ $\mathrm{kg}$ body weight) and the chest was split and the heart tissue was collected. In order to measure the indices, the nitrogen fluid was applied for powdering the heart tissue, and then $0.1 \mathrm{~g}(100 \mathrm{mg})$ of the powder was homogenized with $1 \mathrm{~mL}$ of PBS buffer, and then the extracted solution was centrifuged for 15 minutes at a speed of $5000 \mathrm{rpm}$, and its serum was used to measure the indices. ${ }^{18}$ To detect the cardiac index, Caspase- 8 ELISA kit made by American MyBioSource Company (MBS2022115 96 tests) was used applying a quantitative sandwich method (sensitivity of $0.023 \mathrm{ng} / \mathrm{mL}) .{ }^{18}$ Cardiac levels of Bcl-2 were also measured by ELISA kits by MyBioSource Inc (MBS704330) made in the United States using a quantitative sandwich method (sensitivity of $0.65 \mathrm{pg} / \mathrm{ml}$ ). ${ }^{19}$ Cardiac levels of NT-proBNP were also measured by ELISA kits by MyBioSource Inc (MBS2509359) made in the United States using a quantitative sandwich method (sensitivity of $2.49 \mathrm{ng} / \mathrm{L}$ ). All of the above steps were carried out in the Biochemistry Laboratory of the Faculty of Physical Education and Sports Science in Mazandaran University.

\section{Statistical Analysis}

Shapiro-Wilk test was run to measure the normal distribution of data. Regarding the nature of the distribution of data, for comparing the groups in the variables studied, two-way analysis of variance (ANOVA) was used. In addition, a Tukey test was conducted as the post hoc test. The level of significance was $P<0.05$. Statistical procedures were done using SPSS software package version 22.0.

\section{Results}

Figure 1 shows the mean and standard deviation of caspase-8 levels in heart tissues in the present study. As it can be seen, caspase- 8 levels in diabetic rats were significantly higher compared to control group $(P=$ $0.001)$. On the other hand, the results indicated that caspase- 8 levels in the exercise group were significantly lower compared to the diabetes group $(P=0.001)$.
Moreover, the results showed a significant increase of caspase- 8 levels in exercise + diabetes group compared to exercise group $(P=0.001)$.

Figure 2 shows the levels of $\mathrm{Bcl}-2$ in various study groups in terms of mean and standard deviation. As it can be seen, there is no significant difference between the groups.

Figure 3 shows the mean and standard deviation of NT-proBNP in heart tissue of different groups in the current study. Our results indicated that NT-proBNP in the diabetes groups was significantly higher compared to the control group $(P=0.001)$. However, NT-proBNP significantly decreased in the exercise group compared to control group $(P=0.001)$. It was also observed that NTproBNP significantly reduced in the exercise and exercise + diabetes compared to the diabetes group $(P=0.001$ and $P=0.014)$.

\section{Discussion}

This study aimed to determine the changes in cardiac levels of caspase-8, Bcl-2 and NT-proBNP, as markers for apoptosis and inhibition of heart apoptosis, in STZinduced diabetic rats after 4 weeks of aerobic exercise

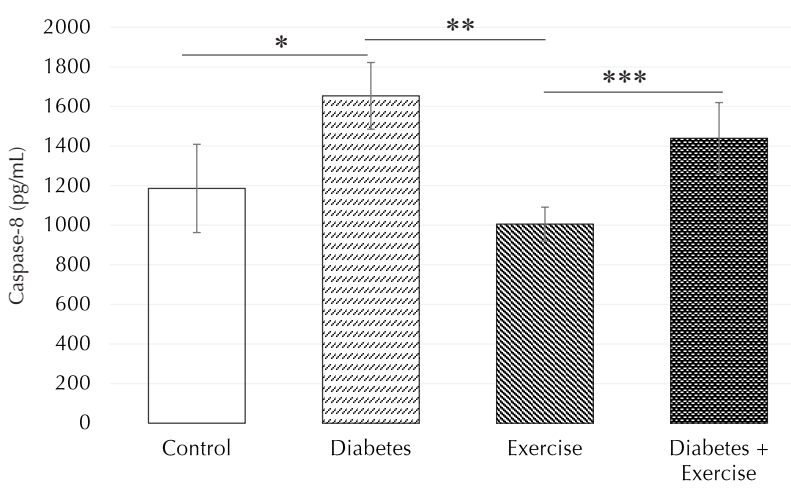

Figure 1. The mean and standard deviation of caspase- 8 in picograms per milliliter. ${ }^{*} P=0.001,{ }^{* *} P=0.001$, and ${ }^{* * *} P=$ 0.014 represent a significant difference between the groups. $P<$ 0.05 was considered as the level of significance.

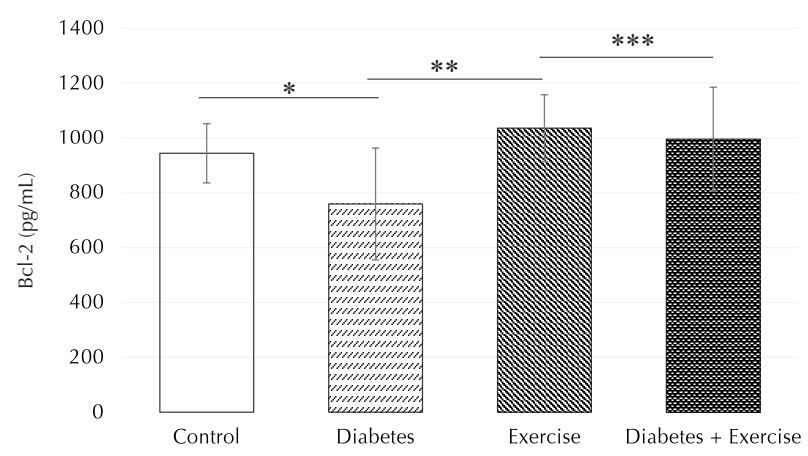

Figure 2. The mean and standard deviation of $\mathrm{BCl}-2$ in picograms per milliliter. $* P=0.001,{ }^{* *} P=0.001$ and $* * * P=0.014$ represent a significant difference between the groups. $P<0.05$ was considered as the level of significance. 


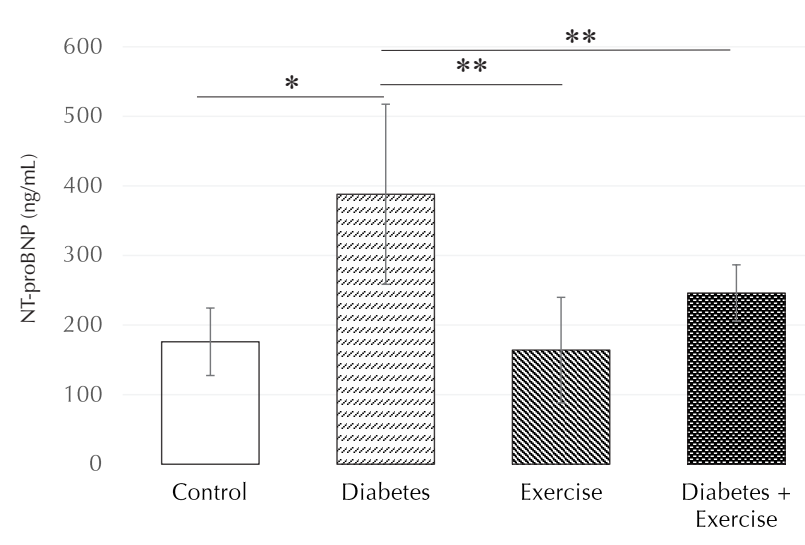

Figure 3. The mean and standard deviation of NT-proBNP in terms of nanograms per milliliter. ${ }^{*}$ and ${ }^{* *} P=0.001$ indicates a significant difference between the groups. $P<0.05$ was considered as the level of significance.

(running). The results revealed that intraperitoneal injection of STZ (55 mg/kg body weight) resulted in a significant increase in caspase-8, and NT-proBNP levels and no significant changes in Bcl-2 levels. Diabetes is the most prevalent metabolic disorder in the developing world. Extensive studies are underway to find suitable treatments for diabetes. ${ }^{20}$

Similarly, Kanter et al reported histological disorders including loss of muscle fibers and irregularities in striated muscles after induction of diabetes. ${ }^{16}$ Moreover, Shamsaei et al observed that after induction of diabetes in rats, necrosis and apoptosis occurred in the neurons of the hippocampus. ${ }^{30} \mathrm{Kim}$ et $\mathrm{al},{ }^{31}$ in contrast, showed that caspase- 3 increased in the eyes of diabetic rats. In addition, the expression of pro-apoptotic protein Bax and $\mathrm{Bcl}-2$ increased in the eyes of diabetic rats. The reason for this discrepancy in the change of $\mathrm{Bcl}-2$ protein can be due to differences in the tissue levels and the expression of the protein gene since changes in the level of gene expression vary with the changes in the tissue levels, and these changes are not necessarily associated..$^{22,32}$ Dousar et al also revealed the incidence of apoptosis in myopathy of diabetic rats. ${ }^{33}$ Joussen et al demonstrated the activation of caspase pathways, damage to retinal cells, apoptosis, and endothelial cell loss in diabetic rats, confirming the results of the present study. ${ }^{34}$ Kang et al stated that the phosphorylation of the pro-apoptosis protein Bad decreases, emphasizing the progression of the apoptosis in the mesenchymal cells in high concentrations of glucose. These disorders in the expression and phosphorylation of the Bcl-2 family are associated with releasing of cytochrome $\mathrm{c}$ and the activation of caspase. It was reported that oxidative stress in mesenchymal cells exposed to high concentration of glucose is an important incidence in the activation of the cell death program that results in mitochondrial dysfunctions and activation of caspase- $3 .{ }^{35}$

In line with the current study, Shiroo et a observed severe apoptosis in cardiac cells of untreated diabetic rats. Furthermore, diabetes in the rats significantly increased the lipid peroxidation rate, the levels of carbonyl protein as an index of protein oxidation, and the superoxide dismutase. ${ }^{36}$ Diabetes increases the level of oxidative stress, which leads to elevated levels of reactive oxygen species and reduces the antioxidant defense capacity, resulting in the programmed death of heart cells and apoptosis. ${ }^{21}$ However, precise molecular mechanisms of apoptosis have not yet been determined by high glucose concentrations. Scholars have argued that the mechanism of apoptosis, where glucose induces cell death, varies depending on the cell and tissue studied. ${ }^{16}$ Research has reported that the cause of diabetes-induced cardiac apoptosis, in addition to increased stress or oxidative stress, is the occurrence of inflammatory processes and the presence of cytokines such as TNF- $\alpha$, IL- $1 \beta$, and IFN- $\gamma$. In addition, their effect on nitric oxide causes the appearance of Fas ligand by inflammatory and cardiac cells, which ultimately leads to the activation of caspase signaling and ultimately cell death by apoptosis in cardiac cells. ${ }^{37}$

It was also observed that in non-diabetic groups, exercise performance did not cause any changes in caspase-8, NT-proBNP and Bcl-2. On the other hand, the exercise in diabetic groups led to a significant decrease in NTproBNP and no changes in caspase- 8 and $\mathrm{Bcl}-2$ were observed. Previous studies showed that increased activity of antioxidant enzymes and decreased lipid peroxidation levels which are followed by exercise have important effects on the prevention of complications of apoptosis caused by diabetes and tissue damage caused by oxidative stress following the disease. ${ }^{24}$ Regular exercise activity has been shown to increase the activity of antioxidant enzymes, increase the resistance to oxidative stress, and thus reduce oxidative damage. ${ }^{25}$ Previous evidence has shown that regular exercise is effective in preventing and delaying diabetes, increasing insulin sensitivity and improving glucose metabolism. ${ }^{38}$ It has also been shown that exercise before ischemia results in a decrease in the ratio of pro-apoptotic proteins and anti-apoptotic proteins, such as Bcl-2, and decreased signaling of caspase pathway activation, especially caspase- 3 (final caspase of apoptosis pathway). ${ }^{39}$ The inhibitory capability of free radicals is probably one of the most important mechanisms in the field of cell defense against cardiac damage. Active oxygen species in the mitochondrial electron transfer chain are produced as a natural product; however, when their level exceeds the antioxidant capacity of the cell, they can lead to cell death. Oxidative stress induced by active oxygen species is highly associated with diabetes and its complications and can cause cell death through various pathways. $^{39}$

NT-proBNP is a precursor of the BNP hormone with 108 amino acids that is broken down by a protease series into 2 CT-BNP molecules with 77 to 108 amino acids and NT-proBNP with 1 to 76 amino acids after 
production and release from the left ventricle. Research has shown that BNP and NT-proBNP levels increase in ventricular hypertrophy and left ventricular dysfunction, atrial fibrillation, severe hypertension, congestive heart failure, myocardial infarction, pulmonary hypertension, pregnancy and chronic renal failure hypertension, and metabolic abnormalities such as diabetes. It has been determined that NT-proBNP enjoys a very high diagnostic value of cell wall stress due to higher halflife. This is consistent with the results obtained in this research. In previous studies, it has also been observed that running exercise reduces the levels of NT-proBNP. These results are consistent with the current research. Cellular and molecular factors are associated with each other through cascade signaling. Following external stimulus and stress, this intercalating cascade of signaling occurs. Protein kinase B is the main agent in the signaling pathway of phosphatidylinositol-3 kinase, which plays a role in many cellular processes, including cellular survival, metabolism, cell growth and proliferation. Increasing the expression and enhancement of protein kinase B activity inhibit apoptosis pathways by phosphorylation of the antiapoptotic proteins of the Bcl-2 family and inactivating the apoptotic precursor protein such as Bax or by directly controlling caspase activity. ${ }^{31}$ Studies have reported that levels of protein kinase B decrease in animal samples with diabetes mellitus. ${ }^{31}$ Furthermore, probably another mechanism of cellular protection from sporadic exercises against apoptosis is associated with the important effects of exercise in enhancing the expression of protein kinase $\mathrm{B}$ since it has been shown that protein kinase B encounters an increase in aerobic exercise. ${ }^{40}$

\section{Conclusion}

Finally, it can be concluded that 4 weeks of aerobic exercise probably reduces the severity of apoptosis in diabetic rats.

\section{Competing Interests}

Authors declare that they have no competing interests.

\section{Ethical Approval}

All the ethics of work with animals were examined by the Ethics Committee of Razi University of Kermanshah and approved with code 024-2-396.

\section{Acknowledgments}

The present study was extracted from the Ph.D. dissertation in Exercise Physiology in the field of cardiovascular, vascular and respiratory diseases. All the officials and those who helped us carry out this research are hereby highly appreciated.

\section{References}

1. Gill C, Mestril R, Samali A. Losing heart: the role of apoptosis in heart disease--a novel therapeutic target? Faseb j. 2002;16(2):135-146. doi:10.1096/fj.01-0629com
2. Narula J, Haider N, Virmani R, et al. Apoptosis in myocytes in end-stage heart failure. N Engl J Med. 1996;335(16):11821189. doi:10.1056/nejm199610173351603

3. Ernande L, Derumeaux G. Diabetic cardiomyopathy: myth or reality? Arch Cardiovasc Dis. 2012;105(4):218-225. doi:10.1016/j.acvd.2011.11.007

4. Fuentes-Antras J, Picatoste B, Gomez-Hernandez A, Egido J, Tunon J, Lorenzo O. Updating experimental models of diabetic cardiomyopathy. J Diabetes Res. 2015;2015:656795. doi:10.1155/2015/656795

5. Shen E, Li Y, Li Y, et al. Racl is required for cardiomyocyte apoptosis during hyperglycemia. Diabetes. 2009;58(10):2386-2395. doi:10.2337/db08-0617

6. Marzetti E, Privitera G, Simili V, et al. Multiple pathways to the same end: mechanisms of myonuclear apoptosis in sarcopenia of aging. ScientificWorldJournal. 2010;10:340349. doi:10.1100/tsw.2010.27

7. Tamm I, Schriever F, Dörken B. Apoptosis: implications of basic research for clinical oncology. Lancet Oncol. 2001;2(1):33-42. doi:10.1016/S1470-2045(00)00193-5

8. Scott I. The role of mitochondria in the mammalian antiviral defense system. Mitochondrion. 2010;10(4):316320. doi:10.1016/j.mito.2010.02.005

9. Suen DF, Norris KL, Youle RJ. Mitochondrial dynamics and apoptosis. Genes Dev. 2008;22(12):1577-1590. doi:10.1101/ gad. 1658508

10. Talanian RV, Quinlan C, Trautz S, et al. Substrate specificities of caspase family proteases. J Biol Chem. 1997;272(15):9677-9682.

11. Kim R, Emi M, Tanabe K, Murakami S, Uchida Y, Arihiro K. Regulation and interplay of apoptotic and non-apoptotic cell death. J Pathol. 2006;208(3):319-326. doi:10.1002/ path. 1885

12. Jiang $\mathrm{X}$, Wang X. Cytochrome C-mediated apoptosis. Annu Rev Biochem. 2004;73:87-106. doi:10.1146/annurev. biochem.73.011303.073706

13. Li H, Zhu H, Xu CJ, Yuan J. Cleavage of BID by caspase 8 mediates the mitochondrial damage in the Fas pathway of apoptosis. Cell. 1998;94(4):491-501. doi:10.1016/S00928674(00)81590-1

14. Arroba AI, Frago LM, Argente J, Chowen JA. Activation of caspase 8 in the pituitaries of streptozotocin-induced diabetic rats: implication in increased apoptosis of lactotrophs. Endocrinology. 2005;146(10):4417-4424. doi:10.1210/en.2005-0517

15. Wright KJ, Thomas MM, Betik AC, Belke D, Hepple RT. Exercise training initiated in late middle age attenuates cardiac fibrosis and advanced glycation end-product accumulation in senescent rats. Exp Gerontol. 2014;50:918. doi:10.1016/j.exger.2013.11.006

16. Kanter M, Aksu F, Takir M, Kostek O, Kanter B, Oymagil A. Effects of Low Intensity Exercise Against Apoptosis and Oxidative Stress in Streptozotocin-induced Diabetic Rat Heart. Exp Clin Endocrinol Diabetes. 2017;125(9):583591. doi:10.1055/s-0035-1569332

17. Bugger $H$, Abel ED. Rodent models of diabetic cardiomyopathy. Dis Model Mech. 2009;2(9-10):454-466. doi:10.1242/dmm.001941

18. Buzas K, Megyeri K, Hogye M, Csanady M, Bogats G, Mandi Y. Comparative study of the roles of cytokines and apoptosis in dilated and hypertrophic cardiomyopathies. 
Eur Cytokine Netw. 2004;15(1):53-59.

19. Kwak HB, Lee Y, Kim JH, Van Remmen H, Richardson AG, Lawler JM. MnSOD overexpression reduces fibrosis and pro-apoptotic signaling in the aging mouse heart. J Gerontol A Biol Sci Med Sci. 2015;70(5):533-544. doi:10.1093/gerona/glu090

20. Baily C, Flatt P. Antidiabetic drugs, new developments. Indian Biotech. 1986;6:139-42..

21. Wang J, Song Y, Wang Q, Kralik PM, Epstein PN. Causes and characteristics of diabetic cardiomyopathy. Rev Diabet Stud. 2006;3(3):108-117. doi:10.1900/rds.2006.3.108

22. Kendrick N. A gene's mRNA level does not usually predict its protein level. Madison: Kendricklabscom; 2014.

23. Cao JY, Wang H. Role of Fas-FasL in insulitis in nonobese diabetic mouse. Chin Med J (Engl). 2004;117(4):615-617.

24. Mohammadi M, Salehi I, Farajnia S. Effect of swimming exercise on oxidative stress in hippocampus of diabetic male rats. Medical Journal of Tabriz University of Medical. 2008;30(2):111-118. [Persian].

25. Mazzola PN, Terra M, Rosa AP, et al. Regular exercise prevents oxidative stress in the brain of hyperphenylalaninemic rats. Metab Brain Dis. 2011;26(4):291-297. doi:10.1007/s11011-011-9264-8

26. Chow SC, Wang H, Shao J. Sample Size Calculations in Clinical Research. CRC press; 2007.

27. Shirinbayan V, Roshan VD. Pretreatment effect of running exercise on HSP70 and DOX-induced cardiotoxicity. Asian Pac J Cancer Prev. 2012;13(11):5849-5855.

28. Shirinbayan V, Dabidi Roshan V, Mahjoub S. The Therapeutic Effect of Endurance Training on Adriamycininduced Cardiac Stress in Rats. Iranian Journal Health Physical Activity. 2013;4(2):8-17.

29. Kavanagh K, Flynn DM, Nelson C, Zhang L, Wagner JD. Characterization and validation of a streptozotocininduced diabetes model in the vervet monkey. J Pharmacol Toxicol Methods. 2011;63(3):296-303. doi:10.1016/j. vascn.2011.02.003

30. Shamsaei N, Abdi H, Shamsi M. The Effect of a Continuous Training on Necrosis and Apoptosis Changes in the
Hippocampus of Diabetic Rats. Journal of Ilam University of Medical Sciences. 2017;25(1):1-11. [Persian].

31. Kim DY, Jung SY, Kim CJ, Sung YH, Kim JD. Treadmill exercise ameliorates apoptotic cell death in the retinas of diabetic rats. Mol Med Rep. 2013;7(6):1745-1750. doi: 10.3892/mmr.2013.1439

32. Alberts B, Johnson A, Lewis J, Walter P, Raff M, Roberts K. Molecular Biology of the Cell. 4th ed. ew York: Garland Science.; 2002.

33. Doustar Y, Mohajeri D, Rezaei A, Hashemi M. The role of endurance swimming on the occurrence of apoptosis in experimental myopathy of diabetic rat. Vet Clin Pathol. 2009;3(4):629-36.

34. Joussen AM, Doehmen S, Le ML, et al. TNF-alpha mediated apoptosis plays an important role in the development of early diabetic retinopathy and long-term histopathological alterations. Mol Vis. 2009;15:1418-1428.

35. Kang BP, Frencher S, Reddy V, Kessler A, Malhotra A, Meggs LG. High glucose promotes mesangial cell apoptosis by oxidant-dependent mechanism. Am J Physiol Renal Physiol. 2003;284(3):F455-466. doi:10.1152/ ajprenal.00137.2002

36. Shiroo A, Salami S, Khadem Ansari M, et al. Protective Effect of Vitamin E on Diabetes Induced Apoptosis and Oxidative Stress in Rat Heart Tissue. Iranian Journal Endocrinology Metabolism. 2008;10(1):67-74.

37. Cao JY, Wang H. Role of Fas-FasL in insulitis in nonobese diabetic mouse. Chin Med J (Engl). 2004;117(4):615-617.

38. Derouich M, Boutayeb A. The effect of physical exercise on the dynamics of glucose and insulin. J Biomech. 2002;35(7):911-917.

39. Hong JH, Kim MJ, Park MR, et al. Effects of vitamin E on oxidative stress and membrane fluidity in brain of streptozotocin-induced diabetic rats. Clin Chim Acta. 2004;340(1-2):107-115.

40. Libonati JR. Cardiac Effects of Exercise Training in Hypertension. ISRN Hypertension. 2013; 2013. doi: $10.5402 / 2013 / 980824$. 